\title{
GENERALIZED PARTON DISTRIBUTIONS AT LARGE MOMENTUM TRANSFER ${ }^{a}$
}

\author{
P. KROLL \\ Fachbereich Physik, Universität Wuppertal, D-42097 Wuppertal, Germany \\ E-mail: kroll@theorie.physik.uni-wuppertal.de
}

\begin{abstract}
The role of generalized parton distributions in wide-angle exclusive reactions will be discussed. In contrast to deep virtual exclusive reactions the wide angle processes offer the possibility of investigating the generalized parton distributions at large momentum transfer.
\end{abstract}

\section{Introduction}

Recently, deep virtual exclusive reactions such as Compton scattering or hard meson electroproduction at large photon virtualities, $Q^{2}$, and small momentum transfer, $-t$, attracted much interest. One can show 1 . 3 that the relevant amplitudes factorize in parton-level amplitudes and process independent soft proton matrix elements which represent generalized parton distributions (GPDs), see Fig. 1. One may also consider Compton scattering or meson electroproduction in the wide-angle region where $-t$ (and $-u$ ) is large but $Q^{2}$ small. These processes are controlled by the handbag diagram shown in Fig. 11 to $\rho$ and their amplitudes also factorize in parton-level amplitudes and GPDs 5. T. The analyses of deep virtual and wide-angle reactions provide complementary informations on GPDs, namely either on their small $-t$ or on their large $-t$ behaviour, respectively. In this talk I am going to report on studies of the wide-angle exclusive reactions.
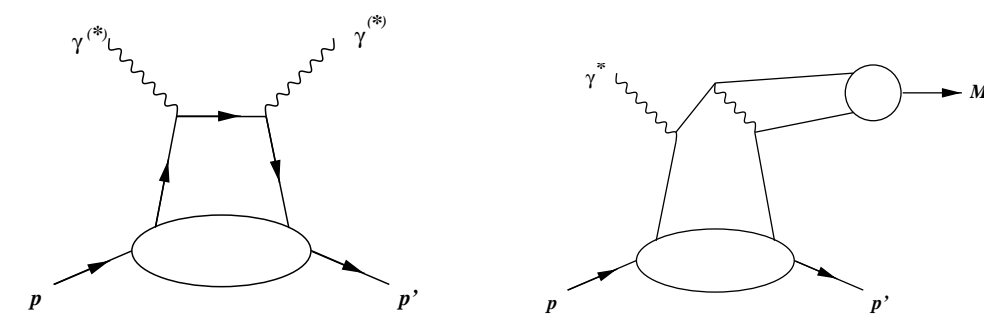

Figure 1: The handbag diagram for Compton scattering (left) and for meson electroproduction (right).

${ }^{a}$ Talk presented at the 9th Intern. Workshop on Deep Inelastic Scattering, Bologna(2001). 

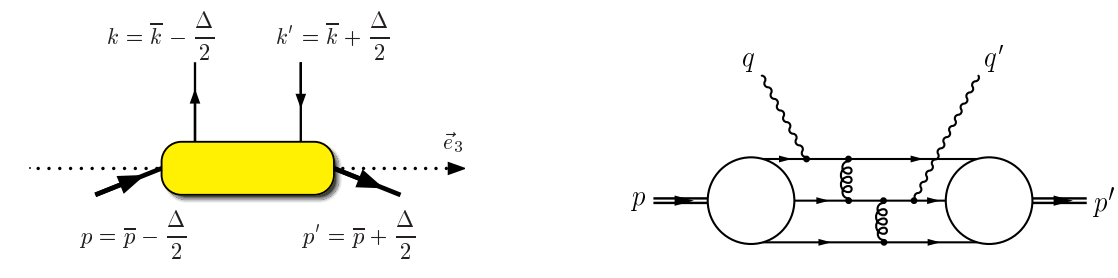

Figure 2: The kinematics for GPDs (left) and a typical Feynman graph for Compton scattering at asymptotically large $-t$.

\section{GPDs and soft physics}

The GPDs $H^{q}(\bar{x}, \xi ; t)\left(\widetilde{H}^{q}\right)$ and $E^{q}(\bar{x}, \xi ; t)\left(\widetilde{E}^{q}\right)$ for a quark of flavour $q$ are defined $\left(\gamma^{+} \gamma_{5}\right)$ 此. The fractions of light-cone plus momentum components appearing as arguments of the GPDs, are given by (see Fig. 2)

$$
\xi=\frac{\left(p-p^{\prime}\right)^{+}}{\left(p+p^{\prime}\right)^{+}}, \quad \bar{x}=\frac{\left(k+k^{\prime}\right)^{+}}{\left(p+p^{\prime}\right)^{+}} .
$$

The GPDs describe the emission of a quark with momentum fraction $(\bar{x}+$ $\xi) /(1+\xi)$ from the proton and the reabsorption of a quark with momentum fraction $(\bar{x}-\xi) /(1-\xi)$. Reduction formulas relate the GPDs to the ordinary parton distributions:

$$
H^{q}(\bar{x}, 0 ; 0)=q(\bar{x}), \quad \widetilde{H}^{q}(\bar{x}, 0 ; 0)=\Delta q(\bar{x}) .
$$

Integrating the GPDs over $\bar{x}$, one obtains the contributions of flavour $q$ quarks to the proton form factors, e.g.,

$$
F_{1}^{q}(t)=\left\langle\bar{x}^{0}\right\rangle=\int_{-1}^{1} d \bar{x} H^{q}(\bar{x}, \xi ; t)
$$

Multiplying $F_{1}^{q}$ by the appropriate electric charges and summing over all flavours, one obtains the full Dirac form factor. The $\left\langle\bar{x}^{0}\right\rangle$-moments $E^{q}$ provides contributions to the Pauli form factor $F_{2}$.

As is well-known, for asymptotically large momentum transfer wide-angle exclusive scattering is controlled by perturbative contributions where all partons the proton is made off, participate in the hard scattering, see Fig. 2. There is evidence that the perfurbative contributions to the proton form factor $\mathrm{A}$ and to Compton scattering 6 are way below experiment provided plausible proton wave functions are used. The soft handbag contributions depicted in Fig. 目, 
seem to dominate at finite values of momentum transfer 5,10 despite of the fact that they formally represent power corrections to the perturbative ones. The soft contributions are defined 5 through the assumption that the (soft) light-cone wave functions (LCWFs) occuring in the Fock decomposition of the proton, are dominated by parton virtualities $k_{i}^{2}, k_{i}^{\prime 2} \lesssim \Lambda^{2}$ and by intrinsic transverse momenta that satisfy $\tilde{k}_{\perp i}^{2} / \tilde{x}_{i}, \hat{k}_{\perp i}^{\prime 2} / \hat{x}_{i}^{\prime} \lesssim \Lambda^{2}$ where $\Lambda$ represents a typical hadronic scale of order $1 \mathrm{GeV}$. Using a symmetric frame in which $\Delta^{+}=\xi=0$, one can show that the active partons, i.e. those to which the photons couple (see Fig. 1), are approximately on-shell, collinear with their parent hadrons and carry momentum fractions close to unity. Thus, the physical situation is that of a hard photon-parton scattering and a soft emission and re-absorption of partons by the protons. In this situation the hadronic amplitudes factorize into parton-level amplitudes (either $\gamma^{*} q \rightarrow \gamma q$ or $\gamma^{*} q \rightarrow M q$ ) and $1 / \bar{x}$-moments of $\xi=0$ GPDs. The proton helicity conserving Compton amplitudes read

$$
\begin{aligned}
\mathcal{M}_{\mu^{\prime}+, \mu+}(s, t)=2 \pi \alpha_{\mathrm{em}} & {\left[\mathcal{H}_{\mu^{\prime}+, \mu+}(s, t)\left(R_{V}(t)+R_{A}(t)\right)\right.} \\
& \left.+\mathcal{H}_{\mu^{\prime}-, \mu-}(s, t)\left(R_{V}(t)-R_{A}(t)\right)\right] .
\end{aligned}
$$

$\mu\left(\mu^{\prime}\right)$ denotes the helicity of the incoming (outgoing) photon. The $\mathcal{H}_{\mu^{\prime} \nu, \mu \nu}$ denote the familiar amplitudes for Compton scattering off massless quarks (with helicity $\nu$ ). The soft form factors, $R_{V}$ and $R_{A}$, for active quarks of flavour $q$, are given by

$$
R_{V}^{q}(t)=\int_{-1}^{1} \frac{d \bar{x}}{\bar{x}} H^{q}(\bar{x}, 0 ; t), \quad R_{A}^{q}(t)=\int_{-1}^{1} \frac{d \bar{x}}{\bar{x}} \operatorname{sign}(\bar{x}) \widetilde{H}^{q}(\bar{x}, 0 ; t) .
$$

The full form factors are specific to the process under consideration. All charged partons contribute to Compton scattering, (e.g. $\left.R_{V}(t)=\sum e_{q}^{2} R_{V}^{q}(t)\right)$ while in electroproduction the meson selects its valence quarks from the proton. The amplitudes for wide-angle photo- and electroproduction of mesons have a representation similar to (4) 6 .

In principle there is third form factor, $R_{T}$, which represents a $1 / \bar{x}$-moment of the GPD $E^{q}$. Note that $E^{q}$ involves parton orbital angular momentum in an essential way. On the basis of the overlap representation of the GPDs 11 one can argue that $R_{T} / R_{V} \simeq F_{2} / F_{1}$ at large $-t$. Since the SLAC data on $F_{2}$ 12 indicate a behaviour $F_{2} / F_{1} \simeq-m^{2} / t$ and given that the evaluation of the handbag diagram is only accurate up to corfections in $\Lambda^{2} / t, R_{T}$ and, hence, proton helicity flip have been neglected in 5,5 for consistency. The new Jlab data on $F_{2} 13$, however, seems to be compatible with a $m / \sqrt{-t}$ behaviour of the ratio of form factors rather than $-m^{2} / t$. Provided this result will be confirmed, $R_{T}$ is to be taken into account. Note that a behaviour $\propto m / \sqrt{-t}$ for the ratio 
of form factors appears quite natural in the overlap representation (see also Ralston's talk 14 ).

\section{Predictions for Compton scattering}

In order to predict wide-angle exclusive reactions a model for the GPDs is required. It has been shown on the basis of light-cone quantisation 11 that the GPDs possess a representation in terms of LCWF overlaps. It allows to construct a simple model for the GPDs by parameterizing the transverse momentum dependence of the LCWFs as

$$
\Psi_{N} \propto \exp \left[-a_{N}^{2} \sum_{i=1}^{N} k_{\perp i}^{2} / x_{i}\right],
$$

which is in line with the central assumption of the soft physics approach of restricted $k_{\perp i}^{2} / x_{i}$, necessary to achieve the factorization of the amplitudes into soft and hard parts. Without explicit specification of the $x$-dependence of the LCWFs one can then evaluate the $\xi=0$ GPDs from the overlap representation if a common transverse size parameter $a=a_{N}$ is used. One obtains

$$
H^{q}(\bar{x}, 0 ; t)=\exp \left[a^{2} t \frac{1-\bar{x}}{2 \bar{x}}\right] q(\bar{x}), \widetilde{H}^{q}(\bar{x}, 0 ; t)=\exp \left[a^{2} t \frac{1-\bar{x}}{2 \bar{x}}\right] \Delta q(\bar{x}) .
$$

Taking the parton distributions from one of the current analyses of deep inelastic lepton-nucleon scattering and using a value of $\simeq 1 \mathrm{GeV}^{-1}$ for the transverse size parameter, one finds reasonable results for the proton and neutron form factors. Improvements can be obtained by treating the lowest Fock states explicitly with specified $x$-dependencies

The amplitude (4) leads to the following Compton cross section

$$
\frac{d \sigma}{d t}=\frac{d \hat{\sigma}}{d t}\left[\frac{1}{2}\left(R_{V}^{2}(t)+R_{A}^{2}(t)\right)-\frac{u s}{s^{2}+u^{2}}\left(R_{V}^{2}(t)-R_{A}^{2}(t)\right)\right],
$$

where $d \hat{\sigma} / d t$ is the Klein-Nishina cross section for Compton scattering off pointlike fermions. The predictions for this cross section are in fair agreement with experiment. The initial state helicity correlation approximately reads

$$
A_{\mathrm{LL}} \simeq \frac{s^{2}-u^{2}}{s^{2}+u^{2}} \frac{R_{A}(t)}{R_{V}(t)} .
$$

Precise data on both observables would permit an extraction of the form factors, $R_{V}$ and $R_{A}$, quite similar to that of the Dirac and Pauli form factors and, 

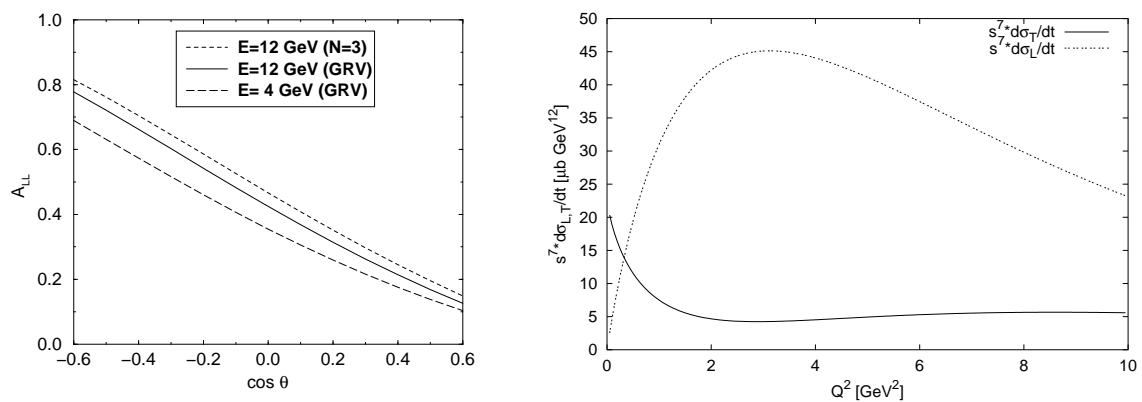

Figure 3: Predictions from the soft physics approach for the helicity correlation $A_{\mathrm{LL}}$ (left) and for the transverse and longitudinal electroproduction cross sections of longitudinally polarised $\rho^{0}$ mesons at $s=40 \mathrm{GeV}^{2}$ and a cm. scattering angle of $90^{\circ}$ (left).

hence, would allow a model independent test of the soft physics approach. Predictions for $A_{L L}$ are shown in Fig. 3. It is to be stressed that $R_{T}$ has been neglected in (8) and (9); its inclusion will lead to corrections of about $10 \%$. The corresponding correlation between the photon helicity and the sideway polarization of the proton (normal to its momentum but in the scattering plane) is particular sensitive to $R_{T}$, namely $A_{L S} / A_{L L} \propto R_{T} / R_{V}$. There are, however, substantial corrections to this result at moderately large values of $-t$.

As an example of wide-angle electroproduction of mesons, the $\rho^{0}$ cross sections for longitudinally and transversally polarised photons are shown in Fig. 3 . The longitudinal cross section dominates except for $Q^{2} \lesssim 1 \mathrm{GeV}^{2}$. Contrary to a statement to be found in the literature occasionally the soft physics approach leads to a $s^{7}$-scaling of the cross sections provided $t / s$ and $Q^{2} / s$ are kept fixed and to the extent that the form factors $R_{V(A)}^{M}$ behave $\propto 1 / t^{2}$. Unfortunately, there is no wide-angle electroproduction data available as yet to compare with. For more details and predictions it is referef to 6 . Predictions for the $e p \rightarrow e p \gamma$ cross sections have also been given in Ref.

\section{Summary}

The GPDs are new tools for the description of soft hadronic matrix elements. They are central elements which connect many different inclusive and exclusive processes: polarised and unpolarised parton distributions are the $\xi=t=0$ limits of GPDs, electromagnetic and Compton form factors represent moments of the GPDs, deeply virtual Compton scattering and hard meson electroproduction are controlled by them. A particularly interesting aspect is touched in exclusive reactions such as proton form factors and wide-angle Compton scat- 
tering. Their analysis by means of GPDs implies the calculation of soft physics contributions to these processes in which only one of the quarks is considered as active while the others act as spectators. The soft contributions formally represent power corrections to the asymptotically leading perturbative contributions in which all quarks participate in the subprocess. There is evidence that for momentum transfers around $10 \mathrm{GeV}^{2}$ the soft contribution dominates over the perturbative one. However, a severe confrontation of this approach with accurate data on wide-angle Compton scattering and electroproduction of mesons is pending.

\section{Acknowledgments}

This work has been supported by the TMR network HPRN-CT-2000-00130.

\section{References}

1. A. V. Radyushkin, Phys. Rev. D 56 (1997) 5524 hep-ph/9704207.

2. X. Ji and J. Osborne, Rev. Rev. D 58, 094018 (1998) [hep-ph/9801260], J. C. Collins and A. Freund, Phys. Rev. D 59, 074009 (1999) [hep$\mathrm{ph} / 9801262$.

3. A. V. Radyushkin, Phys. Lett. B 385, 333 (1996) hep-ph/9605431.

4. X. Ji, Phys. Rev. D 55 (1997) 7114 [hep-ph/9609381].

5. M. Diehl, T. Feldmann, R. Jakob and P. Kroll, Eur. Phys. J. C 8 (1999) 409 hep-ph/9811253 and Phys. Lett. B 460, 204 (1999) hep$\mathrm{ph} / 9903268$.

6. H. W. Huang and P. Kroll, Eur. Phys. J. C 17, 423 (2000) hep$\mathrm{ph} / 0005318$.

7. J. Bolz and P. Kroll, Z. Phys. A 356, 327 (1996) hep-ph/9603289.

8. J. Bolz, R. Jakob, P. Kroll, M. Bergmann and N. G. Stefanis, Z. Phys. C 66, 267 (1995) hep-ph/9405340.

9. T. C. Brooks and L. Dixon, Phys. Rev. D 62, 114021 (2000) hep$\mathrm{ph} / 0004143$.

10. A. V. Radyushkin, Phys. Rev. D 58 (1998) 114008 hep-ph/9803316.

11. M. Diehl et al., Nucl. Phys. B 596, 33 (2001) hep-ph/0009255].

12. L. Andivahis et al., Phys. Rev. D 50, 5491 (1994).

13. M. K. Jones et al. [Jefferson Lab Hall A Collaboration], Phys. Rev. Lett. 84, 1398 (2000) nucl-ex/9910005.

14. J.P. Ralston, P. Jain and R. Buniy, these proceedings and in those of the Conf. of Intersections of Particle and Nuclear Physics, Quebec (2000). 